\title{
COTORRA ARGENTINA (MYIOPSITTA MONACHUS), ESPECIE ANIDANDO CON ÉXITO EN EL SUR DE LA PENÍNSULA DE BAJA CALIFORNIA
}

\author{
ROMEO TINAJERO Y RICARDO RODRÍGUEZ ESTRELLA
}

\author{
${ }^{1}$ Centro de Investigaciones Biológicas del Noroeste (CIBNOR). Instituto Politécnico Nacional No. 195 Col. Playa \\ Palo de Santa Rita Sur, La Paz, Baja California Sur, 23090, México. <estrella@cibnor.mx>
}

Recibido: 03/06/2014; aceptado: 07/04/2015

\begin{abstract}
Tinajero, R. \& Rodríguez Estrella, R. 2015. Cotorra argentina (Myiopsitta monachus), especie anidando con éxito en el sur de la Península de Baja California. Acta Zoológica Mexicana (n. s.), 31(2): 190-197.
\end{abstract}

RESUMEN. Se presenta información sobre la presencia y abundancia así como evidencias de anidación de la cotorra Argentina (Myiopsitta monachus), especie exótica en el sur de la península de Baja California. La cotorra fue registrada por primera vez en noviembre de 2012 en el poblado de Chametla, $7 \mathrm{~km}$ al norte de la ciudad de La Paz. Desde entonces y hasta el día de hoy, el perico ha incrementado su abundancia en 10 veces su población inicial. Reportamos actividad de anidación, entre mayo y agosto de 2013 y mayo -agosto 2014. Los nidos fueron construidos sobre la palma de abanico (Washingtonia robusta, 75\%) y palma de coco (Cocos nucifera, 25\%). Se les observó alimentándose de frutos de palma datilera (Phoenix dactylifera), mezquite (Prosopis sp.), tamarindo (Tamarindus indica), guamúchil (Pithecellobium dulce) y otatave (Vallesia glabra), así como de restos de comida provistos por la gente. Se discuten las posibles implicaciones de la presencia de esta especie exótica en la región.

Palabras clave: Ave exótica, desierto, Baja California Sur, México, Myiopsitta monachus.

\section{INTRODUCCIÓN}

Las especies exóticas pueden causar problemas ecológicos y económicos donde se establecen, sobre todo si se vuelven invasoras. Al establecerse pueden competir por alimento con especies nativas, pueden hibridizarse, transformar y destruir el hábitat de estas especies y pueden ser portadoras de enfermedades y parásitos transmisibles, que inclusive pueden llegar a exterminar poblaciones nativas (Vitouseck et al. 1997, Álvarez-Romero et al. 2008). La Cotorra Argentina (Myiopsitta monacus) es una especie nativa de Sudamérica (Uruguay, Bolivia, Brasil y Argentina) y que se ha reportado recientemente en libertad en diferentes partes de México, como especie exótica y potencialmente invasora (Álvarez-Romero et al. 2008, MacGregor-Fors et al. 2011). La especie ha estado expandiendo su distribución en Europa y Norteamérica, y en algunas regiones se ha registrado un crecimiento exponencial en las zonas donde se ha establecido (Van Bael \& Pruett-Jones 1996, Burger \& Gochfeld 2000, Muñoz \& Real 2006).
Tinajero, R. \& Rodríguez Estrella, R. 2015. Monk Parakeet (Myiopsitta monachus), a new species breeding in the southern part of Baja California. Acta Zoológica Mexicana (n. s.), 31(2): 190197.

ABSTRACT. We present data on presence and abundance, and nesting activity of the exotic monk parakeet (Myiopsitta monachus) in the southern portion of the Baja California peninsula. The monk parakeet was recorded for the first time in November 2012 at the town of Chametla, $7 \mathrm{~km}$ North of La Paz city and since then the monk parakeet has increased its numbers. We recorded nesting activity between MayAugust 2013 and May-August 2014. Nests were built on Mexican fan (Washingtonia robusta, 75\%) and coconut (Cocos nucifera, 25\%) palms. Monk parakeets were observed feeding on fruits of date palm (Phoenix dactylifera), mesquite (Prosopis sp.), tamarin (Tamarindus indica), guamuchil (Pithecellobium dulce) and pearlberry (Vallesia glabra), and remains of human waste foods. Finally, we discuss the potential implications of the establishment of this exotic species in the region.

Key words: Exotic bird, desert scrub, Baja California Sur, Mexico, Myiopsitta monachus.

Esta ave es de tamaño mediano $(29 \mathrm{~cm})$, con plumaje principalmente de color verde, y con el pecho, frente y garganta de color gris; tiene una banda amarillo oliva en el abdomen, cola larga, plumas de sus alas primarias y secundarias de color azul (Fig. 1). La especie no presenta dimorfismo sexual, y los jóvenes, aunque son parecidos a los adultos, difieren por la presencia de plumas verdes entre el plumaje gris de la frente (Alderton \& Stevenson 1991). Se ha introducido en diferentes países de Europa (Portugal, España, Inglaterra, Francia, Alemania, Italia, Bélgica, Republica Checa, Eslovaquia, Dinamarca y Holanda), norte y centro América (Estados Unidos de Norte América, Canadá, México, islas del Caribe), África (Kenia), Asia (Israel y Japón) ( Butler 2002, Hatzofe \& YomTor 2002, Domenech et al. 2003, Pajares 2005, Strubbe \& Matthysen 2009, Mori et al. 2013). La principal forma de introducción ha sido por el comercio activo y el pasivo a través de escapes y liberaciones intencionales. Les favorecen las actividades humanas puesto que se logran establecer con éxito en ciudades y en zonas agrícolas (Muñoz \& Real 2006). En estos ambientes pueden llegar a causar 


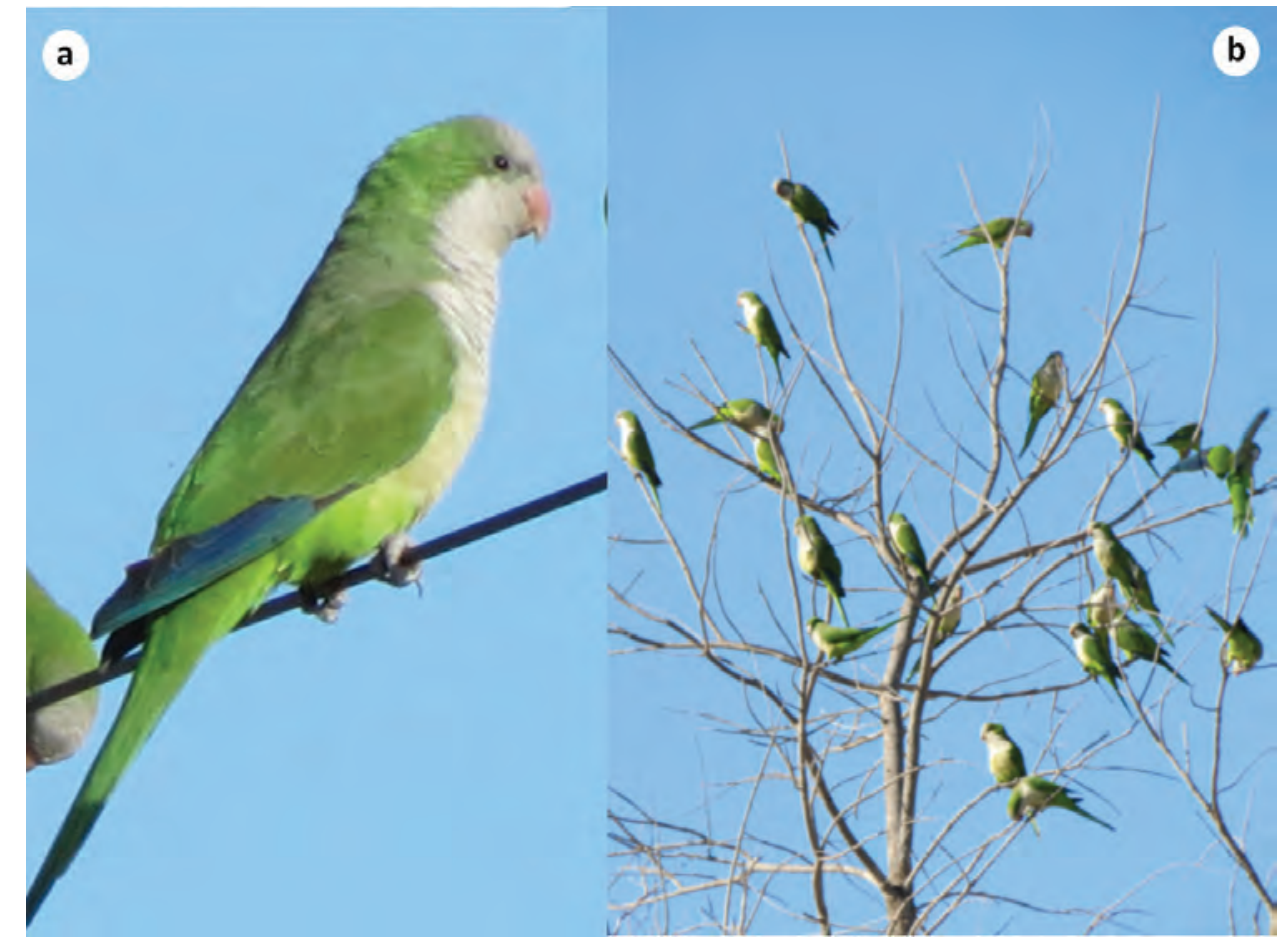

Figura 1. (a) Individuo de cotorra argentina perchado en cables eléctricos. (b) Individuos de cotorra argentina perchados en pino salado (Tamarix spp.). Fotografiados en Chametla, B.C.S.

daños en cultivos anuales (maíz, sorgo, girasol) y árboles frutales (lichi, ojo de dragón, durazno, pera, manzana y cítricos, entre otros) (Freeland 1973, Mott 1973, Tillman et al. 2000, Domenech et al. 2003, Pablo-López 2009); también causa daños a la infraestructura urbana cuando construyen los nidos en subestaciones, torres y postes eléctricos, pudiendo provocar averías al sistema eléctrico (Avery et al. 2006, Pruett-Jones et al. 2007). En el estado de Florida han encontrado que la cotorra argentina causa daños a las huertas con pérdidas millonarias anuales (Tillman et al. 2000). En Cataluña, la especie se ha expandido y ha colonizado algunas zonas de importancia agrícola (Domenech et al. 2003). En México, se ha reportado su presencia en cultivos de sorgo y maíz en el estado de Oaxaca (Pablo-López 2009), pero no se ha hecho una valoración económica de sus efectos.

La cotorra argentina es un ave social, monógama que construye nidos comunales, los cuales son voluminosos y pueden estar constituidos por varias cavidades o cámaras donde diferentes parejas pueden anidar simultáneamente (Navarro el al. 1992). Estas estructuras son utilizadas también como dormitorios durante todo el año (Navarro et al. 1992, Eberhard 1998). Su biología reproductiva ha sido relativamente bien estudiada en su distribución nativa (Navarro \& Bucher 1990, Navarro et al. 1992, Martín \& Bucher 1993, Peris \& Aramburú 1995, Eberhard 1998). En las áreas de invasión este tipo de estudios se han hecho principalmente en los Estados Unidos de Norteamérica, en Chicago (Hyman \& Pruett-Jones 1995), Florida (Avery et al. 2012) y Connecticut (Living 2006). La información es muy escasa en Europa, Asia y África (Batllori \& Nos 1985). Se puede reproducir desde el segundo año de vida (Martín \& Bucher 1993) y el tamaño de la puesta es de 1-12 huevos, con un promedio de 6 (Navarro et al. 1992, Avery et al. 2012). Estas características así como su capacidad para adaptarse a diferentes condiciones ambientales (tropicales, templadas y frías) en diferentes partes del mundo (Simpson \& Ruiz 1974, Hyman \& Pruett-Jones 1995, Strubbe \& Matthysen 2009), la hacen una especie altamente invasora.

Recientemente en México se ha reportado su presencia en libertad en el Distrito Federal, Estado de México, Oaxaca, Querétaro, Michoacán, Puebla, Chiapas, Guanajuato, Chihuahua y en la península de Baja California (Álvarez-Romero et al. 2008, Pablo-López 2009, MacGregor-Fors et al. 2011, Pineda-López \& Malagamba 2011, Ramírez-Albores 2012, Soto-Cruz, et al. 2014). En la península de Baja California ha sido reportada en Mexicali, Ensenada, Guerrero Negro, Loreto y la ciudad de La Paz (MacGregor-Fors et al. 2011, Guerrero-Cárdenas et al. 2012; eBird 2014), pero sólo han sido registros de observaciones, sin un seguimiento sistemático de las poblaciones. En el presente estudio proporcionamos evidencia de la reproducción exitosa de la especie y presenta- 
mos información sobre las variaciones numéricas de una población de cotorra argentina establecida en el sur de la península de Baja California. Además, presentamos información sobre su dieta y estructuras de anidación donde construye sus nidos y discutimos sobre las implicaciones de la reproducción de esta especie para la avifauna de la región.

\section{MATERIAL Y MÉTODOS}

La investigación fue realizada en el poblado de Chametla ubicado a $7 \mathrm{~km}$ al norte de la ciudad de la Paz (2405'55.21” N y 110²2’34.49”' O), en el municipio de La Paz Baja California Sur. Este poblado es relativamente pequeño, con aproximadamente 2,200 habitantes y se encuentra ubicado a una altitud de $5 \mathrm{~m}$ sobre el nivel del mar. El poblado tiene una superficie aproximada de $1 \mathrm{~km}^{2}$ (largo $1.5 \mathrm{~km}$, ancho promedio $0.7 \mathrm{~km}$ ) y está rodeado principalmente de vegetación nativa, que es matorral xerófilo, y una parte colinda con áreas de cultivos. El clima es del tipo Bw(h')hw(x') muy seco con lluvias en verano con una precipitación invernal mayor de 10.2\%. La precipitación media anual es de $171 \mathrm{~mm}$ y la temperatura media anual de $23.4^{\circ} \mathrm{C}$. Las temperaturas máximas que se llegan a presentar en primavera y verano son superiores a $40^{\circ} \mathrm{C}$, inclusive alcanzando los $43^{\circ} \mathrm{C}$ en los meses entre mayo y septiembre. En el invierno, puede haber temperaturas hasta los $37^{\circ} \mathrm{C}$ (INEGI 1994, Ruiz-Corral et al. 2006). Dentro del poblado, la gente ha sembrado palmas nativas, endémicas, como la real o de abanico (Washingtonia robusta), árboles nativos como el mezquite (Prosopis sp.) y el otatave (Vallesia glabra), así como plantas exóticas tales como la palma datilera (Phoenix dactylifera), y el coco (Cocos nucifera), además de distintos árboles como tamarindo (Tamarindus indica), eucaliptos (Eucaliptus spp.), mango (Mangifera indica) y cítricos, entre otros.

La presente investigación se originó a partir de un proyecto de monitoreo de especies exóticas invasoras en el estado de Baja California Sur, el cual se ha desarrollado desde hace alrededor de 10 años. El primer registro de la cotorra argentina en el poblado de Chametla y en la zona se hizo en el mes de noviembre de 2012, cuando se contabilizó en varias ocasiones un grupo de 10 aves (T. López \& A. Martínez, com. pers.). Antes de esta fecha no hay registros de la especie a pesar de ser un sitio de censado de aves. Debido a su ruidosa actividad, se les podía seguir y ubicar prácticamente sin problema. A partir de mayo de 2013, se inician los muestreos de las cotorras con un protocolo de seguimiento sistemático con una duración de un año. En las áreas donde se registró su presencia se contabilizó el número total de individuos observados, su actividad, la percha utilizada y el tipo de alimento que con- sumían. Se hicieron los conteos de individuos únicamente en las áreas de anidación, sobre todo a partir de enero de 2014 con un método corregido en función de las observaciones previas, con el fin de estandarizar los números y tener una mejor estima del tamaño de la población, ya que se sabe que estas aves utilizan los nidos como dormideros comunales. De esta manera, en los sitios de anidación se realizó una vigilancia con binoculares registrando la llegada y salida de cotorras en los nidos durante dos horas antes de que oscureciera (Belinchón-Navarro et al. 1999). La búsqueda de nidos se realizó de manera continua cada mes, recorriendo todo el poblado a pie y en vehículo, observando árboles, palmas y estructuras como postes y torres eléctricas. Se sabe que a un nido ya preexistente se le pueden añadir otras cámaras de anidación, conformándose de esta manera un nido compuesto que consta de varias cámaras independientes (Navarro et al. 1992). Una vez que ubicamos los primeros nidos, les dimos seguimiento para ver si construían nuevos anexos o nidos cercanos. Se registraron las estructuras donde construyeron los nidos, y se midió la altura de la planta soporte, el número de cámaras y la cercanía entre nidos. Es importante señalar que debido al relativo pequeño tamaño del poblado, a los estudios previos hechos en la zona y el seguimiento de aves en general sin haber detectado a la especie sino hasta 2012, y debido a lo fácil que es observar y ubicar a los individuos y parvadas de la cotorra argentina, consideramos que nuestro estudio abordó el proceso inicial de invasión de la especie en esta zona y que nuestras observaciones muestran la tendencia de la población en el tiempo.

\section{RESULTADOS}

Abundancia. Nuestros registros para 2013 iniciaron el 28 de mayo, a las 9:00 h, cuando tres individuos se observaron posados en un árbol de tamarindo. El 21 de junio, a las 19:35 h se observaron 20 cotorras, 14 perchadas sobre árboles (8 en tamarindo, 4 en eucalipto, 2 en guamúchil), y seis fueron observadas en vuelo. Consideramos por lo anterior, que el tamaño inicial de la población de la cotorra para nuestros análisis en la zona antes de la temporada reproductiva del 2013 fue de entre 10 y 20 individuos (Fig. 2). Para el 29 de octubre a las 8:10 h se observó una parvada en vuelo constituida por 35 individuos. El 24 de noviembre a las 8:30 h se observó un grupo de 50 individuos perchados en un eucalipto. El 22 de diciembre monitoreamos los nidos a partir de las 8:00 h hasta las 10:50 h, contando la salida de 56 individuos de los nidos. Este día después de salir de los nidos se encontraron tres grupos, uno en cables de luz, otro en plantas de otatave, y 22 individuos perchados en un pino salado (Tamarix spp.) (Fig. 1a, 1b). Finalmente, el 29 de agosto de 2014 registramos 


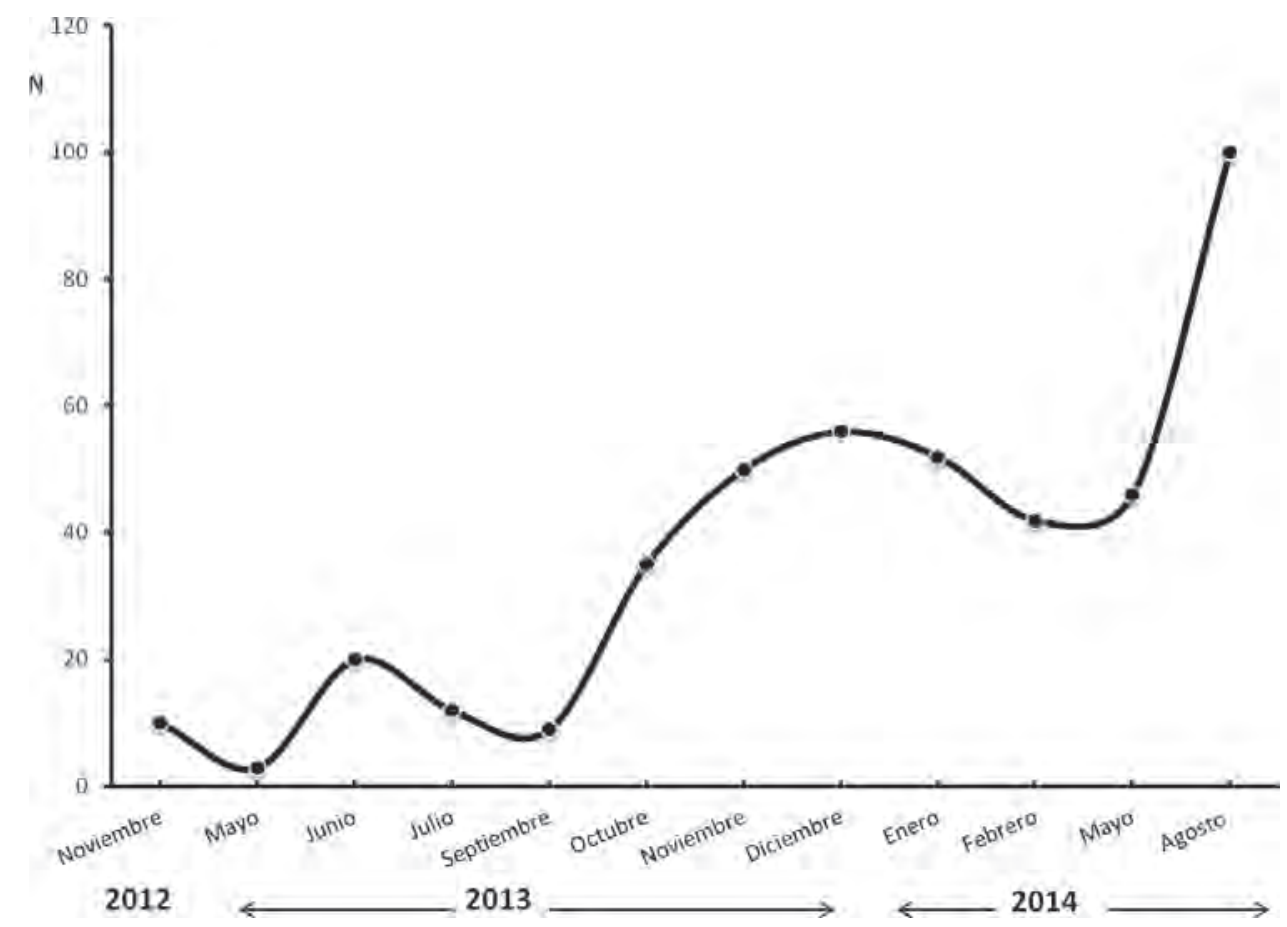

Figura 2. Variación de la abundancia de cotorra argentina (Myiopsitta monachus) durante el periodo de estudio en el poblado de Chametla, B.C.S.

100 individuos que es el número máximo de individuos registrados. Las variaciones de números de cotorras durante los muestreos se presentan en la figura 2.

Reproducción. El primer nido encontrado fue el 28 de mayo de 2013. A las 9:00 h se observaron tres individuos posados en un árbol de tamarindo. Las aves cortaban ramas del árbol, volando posteriormente con ellas en su pico a una palma real o de abanico. En esta palma se encontraban construyendo una estructura con la forma de su nido típico. Posteriormente, el 6 de junio se ubicaron 3 nidos que se encontraban en tres palmas de abanico; los nidos tenían material verde recién colocado (Fig. 3a). Otros 4 nidos fueron encontrados en fechas posteriores durante las visitas constantes que realizamos a los sitios aledaños a los nidos previamente descritos (Cuadro 1). Las características de los nidos y de las estructuras donde se colocaron se presentan en la Cuadro 1. Las cotorras colocaron sus nidos principalmente en palma Washingtonia (75\%) y en palma de coco (25\%) ( $\mathrm{N}=8$ nidos), con una altura promedio de $12.5 \mathrm{~m}$ (+3.3). Los nidos encontrados están muy próximos entre ellos, cuatro con menos de 10 m de separación entre ellos, y el nido más alejado se ubicó a $170 \mathrm{~m}$ de distancia.

Durante las observaciones, se registraron otras actividades reproductivas como apareamiento y cuidados parentales. El 1 de julio a las 18:40 h dos individuos se apareaban sobre un árbol de tamarindo. El 14 de septiembre a las 18:30 h se registraron nueve individuos que vi- sitaban de manera continua uno de los nidos ubicados en una de las palmas de abanico. Seguidamente se hicieron observaciones focales de una hora en un nido que tenía cuatro entradas, observando a los adultos entrando y saliendo en varias ocasiones; registramos en dos entradas del nido dos pollos en cada una (Figura 3 b,c). Observamos que los pollos eran alimentados por los adultos. El 22 de septiembre a las 9:00 h se registró una parvada de seis individuos sobre un mezquite, observando que una cotorra alimentaba a un volantón en el pico. El 19 de mayo de 2014 observamos una pareja con comportamiento de cortejo.

Alimentación. Observamos a las cotorras alimentándose de brotes de tamarindo y mezquite, y de frutos de mezquite, huamúchil (Pithecellobium dulce) y otatave. En tres ocasiones distintas se observaron seis individuos alimentándose de restos de alimento dejados por la gente (tortilla dura) en el patio de casas.

\section{DISCUSIÓN}

Confirmamos por primera vez la reproducción exitosa de la cotorra argentina en la península de Baja California. Aunque previamente se habían mencionado sitios de nidos a la mitad de la península (Guerrero Negro; GuerreroCárdenas et al. 2012), no se mostraron evidencias de que la reproducción de la especie hubiera sido exitosa. La población de la cotorra argentina se ha incrementado fuerte- 


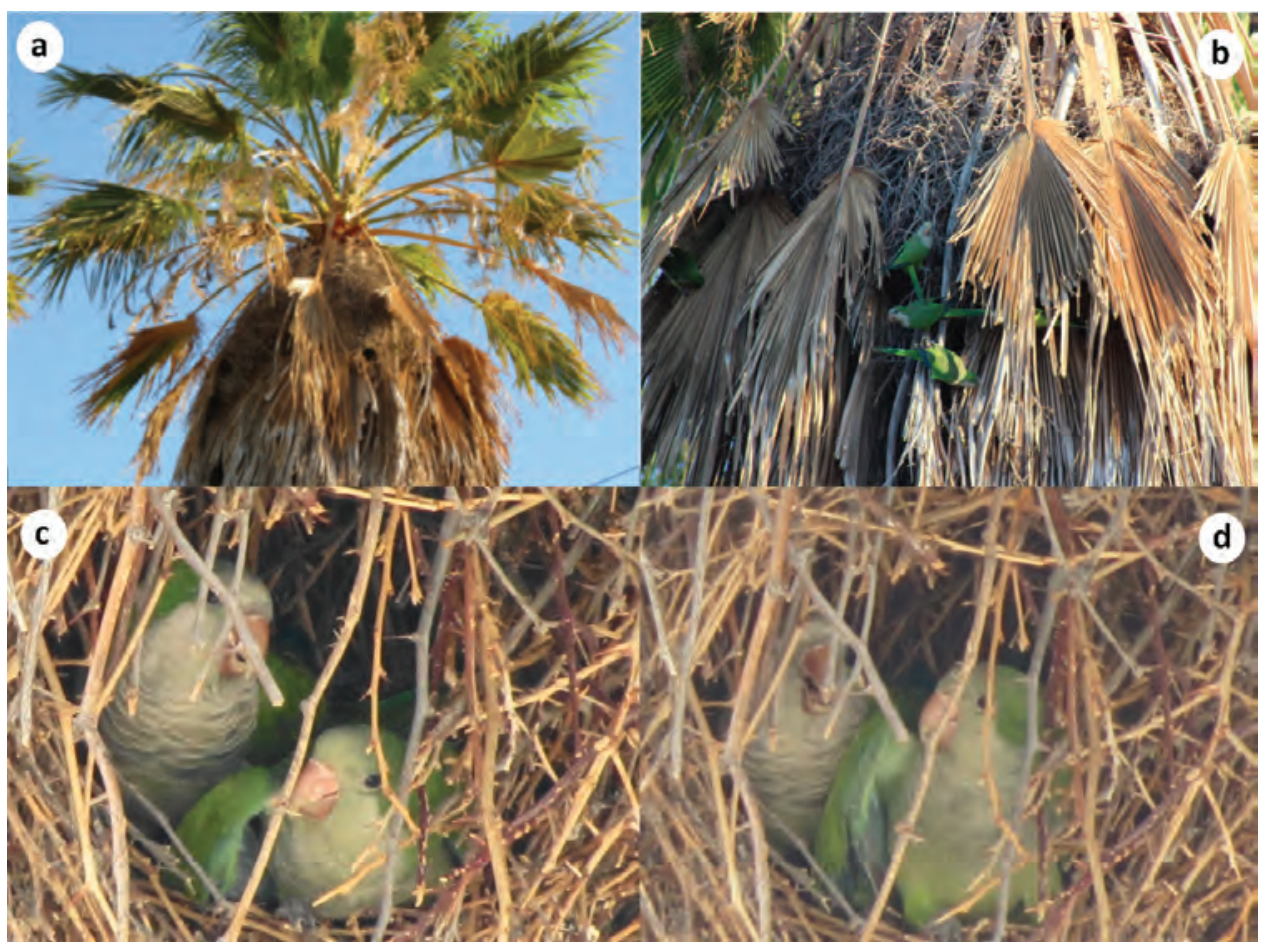

Figura 3. Nidos e individuos de cotorra argentina. (a) Nido construido en palma de abanico. (b) Adultos ingresando a los nidos. (c) Adulto y pollo dentro del nido. Nótese el color del plumaje del pollo, verde oliva sobre frente y pecho, a diferencia del adulto. (d) Pollo en nido demandando alimento (agitando las alas) a su progenitor que estaba a su lado.

Cuadro 1. Características de las estructuras de soporte y de los nidos de la cotorra argentina en Chametla, Baja California Sur, México, 2013-2014.

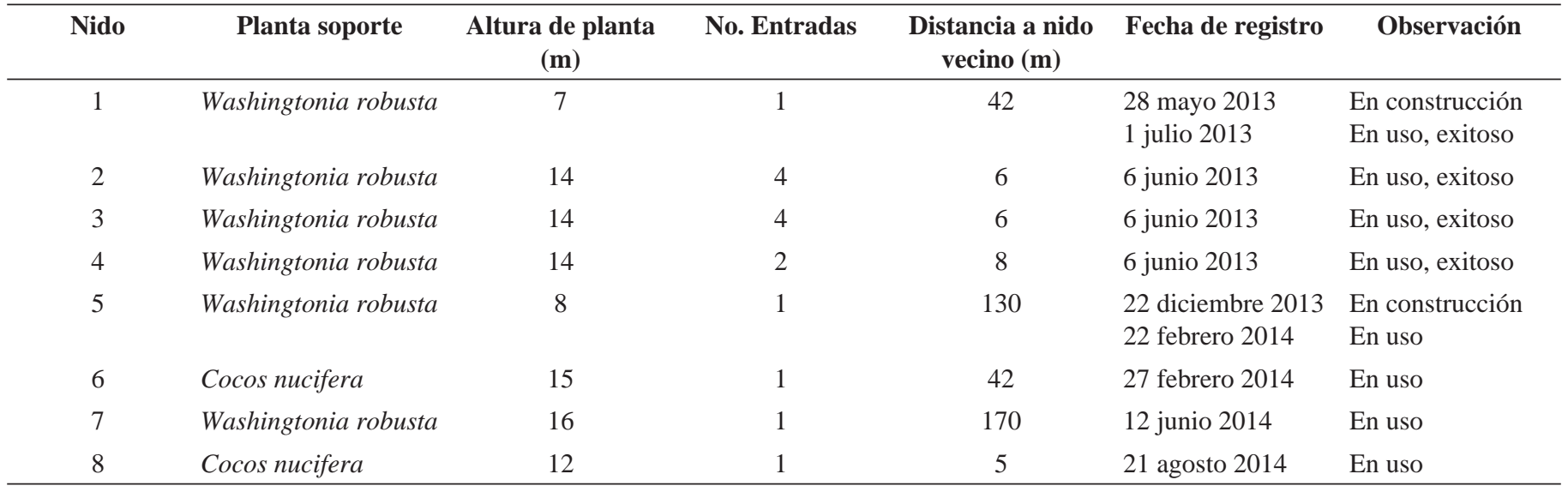

mente en menos de dos años en el poblado de Chametla, así como el número de nidos construidos. En la parte sur de la península de Baja California la cotorra argentina se está reproduciendo en esta fase inicial de invasión, entre mayo y septiembre, ocurriendo el cortejo y construcción de nidos aparentemente en mayo y junio, la incubación en julio, la crianza en agosto y los primeros vuelos de los pollos en agosto-septiembre. Esta época reproductiva es similar a la registrada en su distribución nativa en el hemisferio sur, en Argentina, en las correspondientes estaciones de primavera y verano (Navarro et al. 1992). La época reproductiva que ocurre en esta región de la península difiere de lo registrado en Florida, un área donde es introducida también, donde la reproducción ocurre con unos meses de anterioridad, de marzo a julio (Avery et al. 2012). Hay factores que influyen en el inicio de la época reproductiva de las aves, los cuales aparentemente están relacionados a variables climáticas o de latitud. Por ejemplo, se ha documentado que en la subespecie Myiopsita monachus catita los factores ambientales que influyen en 
el comienzo de las puestas son las temperaturas máximas entre finales de invierno y mediados de primavera (Navarro \& Bucher 1992). Es probable que en La Paz y sus alrededores las temperaturas máximas en estos meses sean similares a lo que se presenta en Argentina mientras que en Florida éstas son más estables, con más calor, por lo que la reproducción puede adelantarse.

Las estructuras donde colocan sus nidos las cotorras en sus áreas de invasión son muy variadas, siendo estructuras artificiales (postes y torres eléctricas) y árboles y palmas (eucaliptos, encinos, palmas). Se ha encontrado en Florida y Chicago que el $80 \%$ y $58 \%$ de los nidos se encuentran en estructuras artifíciales (Avery et al. 2006, Minor et al. 2012), mientras que en España utilizan principalmente palmas Phoenix sp., y en menor medida pinos (Pinus sp.), eucaliptos y álamos (Sol et al. 1997, Domenech et al. 2003); en Cataluña el 78\% de los nidos se encuentran en palmas (Domenech et al. 2003). En nuestro caso, el $100 \%$ de los nidos que encontramos en el poblado de Chametla se construyeron en palmas (Washingtonia robusta y Cocos nocifera).

En áreas de invasión, la cotorra argentina tiene preferencias por sitios urbanos y suburbanos (Hyman \& PruettJones 1995, Muñoz \& Real 2006, Rodríguez-Pastor et al. 2012). En España se ha encontrado que tienen preferencia por barrios con altas densidades de árboles (Pajares 2005). También se ha encontrado que su distribución está determinada principalmente por la actividad humana, sobre todo en zonas urbanas ubicadas en planicies y a orillas de ríos (Muñoz \& Real 2006). En la península de Baja California, sólo se ha reportado a la cotorra en zonas suburbanas (MacGregor-Fors et al. 2011, este estudio). Es notorio que la población de cotorras argentinas del sur de la península de Baja California, Chametla, se encuentra separada $560 \mathrm{~km}$ de la población reportada en Guerrero Negro (Guerrero-Cardenas et al. 2012) y $245 \mathrm{~km}$ del reporte de Loreto (MacGregor-Fors et al. 2011), aunque no hemos confirmado que se encuentre en esta última localidad ni en qué situación está. El 6 de septiembre de 2014, en la ciudad de La Paz registramos un grupo de 14 individuos y cinco nidos, siendo cuatro de ellos activos, registrándose pollos en uno de ellos. Es probable que el de La Paz se trate de un grupo pequeño con menor tiempo de haberse establecido que la población de Chametla, pero dada la cercanía entre poblados podrían considerarse como sub-poblaciones de una misma población. Por lo anterior, no parece que la colonización de Chametla se deba a una ampliación de su distribución por dispersión o migración de la cotorra dentro de la península, ya que la especie no es migratoria y se ha reportado que en su área de distribución natural el rango medio de dispersión de jóvenes de su sitio natal es de 1230 m (Martin \& Bucher
1993); en Barcelona, un sitio donde fue introducida, se ha encontrado una distancia de dispersión muy similar, 1114 m (Carrillo-Ortiz 2009). Aunque estudios genéticos en áreas de invasión en los Estados Unidos de América EUA indican que su dispersión puede llegar a ser de $100 \mathrm{~km}$ (Concalves Da Silva et al. 2010), esto podría funcionar en regiones donde las condiciones ambientales no sean extremas, con ambientes estables. En ambientes áridos extremos como ocurre en la península de Baja California, las probabilidades de viajar y colonizar se reducirían para especies que no son originalmente migratorias. Las zonas urbanas o suburbanas a lo largo de Baja California Sur están separadas unas de otras por grandes distancias y extensiones de desierto de matorral xerófilo que probablemente actúan como barrera para la colonización de sitios adecuados por la cotorra argentina. Por lo anterior, creemos que es el hombre quien ha transportado las cotorras y se han dado liberaciones o escapes posteriores geográficamente separados de cotorras cautivas en estas tres ciudades del sur de la península, tal como se ha reportado ocurre en EUA (Van Bael \& Pruett-Jones 1996). En la ciudad de La Paz se ha observado la venta de cotorra argentina en las calles en los años 2009 y 2010. Recientemente se ha detectado su venta en una tienda de mascotas.

Nuestra predicción es que de no controlar pronto a la población de cotorra argentina en Chametla, se dispersará a poblados próximos como El Centenario y la ciudad de La Paz (ubicados a una distancia menor de 10 km), uniéndose con el grupo de cotorras encontrados en la ciudad de la Paz. Posteriormente podría continuar hacia otros poblados al sur, como el poblado del Triunfo que se encuentra a $40 \mathrm{~km}$, y que es un sitio donde inicia la vegetación de selva baja caducifolia, ambiente tropical, donde hay huertas de naranja, mango, ciruelas y otros. Este poblado parece que podría ofrecer sitios adecuados para que la cotorra se establezca e invada, y continuar más fácilmente la invasión hacia la punta de la península, hacia Los Cabos (véase Muñoz \& Real 2006). Es necesario tomar acciones prontas para controlar las poblaciones de cotorra argentina en libertad. En este momento en que puede iniciar la invasión del sur de la península de Baja California, el método más efectivo para erradicar a la cotorra argentina sería el de disparos y trampeo (Simberloff 2003, Conroy \& Senar 2009). Posteriormente, se complicaría su erradicación y habría que usar inclusive métodos químicos (Yoder et al. 2007, Avery et al. 2008). Recomendamos fuertemente su erradicación en este momento, lo que reducirá costos al futuro. Se debe seguir dando seguimiento a las poblaciones y sitios en tanto la erradicación sucede, así como informar y concientizar a las personas del gobierno y de la sociedad en general sobre los riesgos am- 
bientales que esta especie representa, así como restringir su venta en el país. Nuestras observaciones contribuyen al conocimiento de la distribución de la especie, su ecología, su abundancia, su reproducción, y el tipo de alimento que consumen en un área nueva de potencial invasión, en la península de Baja California.

AGRADECIMIENTOS. Al proyecto SEP-CONACyT (155956) a RRE. RTH recibió una beca posdoctoral durante el desarrollo del trabajo. A José Juan Pérez Navarro por la identificación de plantas. A Tomás López por facilitar la información de las cotorras del año 2012. Dos revisores anónimos hicieron comentarios valiosos.

\section{LITERATURA CITADA}

Alderton, D. \& Stevenson, G. 1991. The atlas of parrots. T.F.H. Publications, Inc. Neptune City, New Jersey. USA.

Álvarez-Romero, J. G., Medellín, R. A., Oliveras de Ita, A., Gómez de Silva, H. \& Sánchez, O. 2008. Animales exóticos en México: una amenaza para la biodiversidad. CONABIO-INECOLUNAM-SEMARNAT. México. D.F.

Avery, M. L., Lindsay J. R., Newman J.R., Pruett-Jones S. \& Tillman, E.A. 2006. Reducing Monk Parakeet impacts to electric utility facilities in south Florida. In: C.J, Feare \& D.P. Cowan (Eds.). Advances in vertebrate pest management.Vol. IV. Filander Verlag Furth. Federal Republic of Germany.

Avery, M. L., Yoder, C. A. \& Tillman, E. A. 2008. Diazacon inhibits reproduction in invasive Monk Parakeet populations. The Journal of Wildlife Management, 72: 1449-1452.

Avery, M. L., Tillman, E.A., Keacher, K. L., Arnett, J. E. \& Lundy, K. J. 2012. Biology of invasive Monk Parakeets in south Florida. The Wilson Journal of Ornithology, 124: 581-588.

Batllori, X. \& Noss, R. 1985. Presencia de la cotorrita gris (Myiopsitta monachus) y de la de collar (Psittacula krameri) en el área metropolitana de Barcelona. Miscelania Zoológica, 9: 407-411.

Belinchón Navarro, C., Grande Pardo, C., Guil López, N., Olmedo Hernández, S., Ruiz Gil, M. P. \& De Juana Aranzana E. 1999. Situación de la cotorra argentina (Myiopsitta monachus) en la ciudad de Madrid. Anuario Ornitológico de Madrid, 1998: 64-71.

Burger, J. \& Gochfeld, M. 2000. Nest site selection in Monk Parakeets (Myiopsitta monachus) in Florida. Bird Behavior, 13: 99-105.

Butler, C. 2002. Breeding parrots in Britain. British Birds, 95: 345-348.

Concalves Da Silva, A., Eberhard, J. R., Wright, T. F., Avery, M. L. \& Russelo, M. A. 2010. Genetic evidence for high propagule pressure and long-distance dispersal in Monk Parakeet (Myiopsitta monachus) invasive population. Molecular Ecology, 19: 33363350.

Conroy, M. J. \& Senar, J. C. 2009. Integration of demographic analyses and decision modeling in support of management of invasive Monk Parakeets, an urban and agricultural pest, pp. 491-510. In: D. L. Thompson, E. C. Cooch \& M. J. Conroy (Eds.). Modeling demographic processes in marked populations. Springer, New York.

Domenech, J., Carrillo, J. \& Senar, J.C. 2003. Population size of the Monk Parakeet Myiopsitta monachus in Catalonia. Revista Catalana de Ornitología, 20: 1-9.

Eberhard, J. R. 1998. Breeding biology of the Monk Parakeet. Wilson Bulletin, 110: 463-473.

eBird. 2014. Una base de datos en línea para la abundancia y distribu- ción de las aves (Aplicación de internet). eBird, Ithaka, New York. Disponible http://www.ebird.org. (Accedido: 22/Enero/2014).

Freeland, D. B. 1973. Some food preferences and aggresive behavior by Monk Parakeets. The Wilson Bulletin, 85: 332-334.

Guerreo-Cárdenas, I., Galina-Tessaro, P., Caraveo-Patiño, J., Tovar-Zamora I., Cruz-Andrés, O.R. \& Álvarez-Cárdenas, S. 2012. Primer registro de la cotorra argentina (Myiopsitta monachus) en Baja California Sur, México. Huitzil, 13: 156-161.

Hatzofe, O. \& Yom-Tor, Y. 2002. Global warming and recent changes in Israel's avifauna. Israel Journal of Zoology, 48: 351-357.

Hyman, J. \& Pruett-Jones, S. 1995. Natural history of the Monk Parakeet in Hyde Park, Chicago. Wilson Bulletin, 107: 510-517.

INEGI. 1994. Síntesis Geográfica del Estado de Baja California Sur. Aguascalientes, México.

Living, S. A. 2006. The Monk Parakeet (Myiopsitta monachus) Boddaert (1783) in Connecticut: An investigation of distribution, nesting ecology, feeding habitats and interactions. M. Sc. Thesis. Southern Connecticut State University, New Haven, Connecticut.

MacGregor-Fors, I., Calderón-Parra, R., Melendez-Herrada, A., López-López, S. \& Schondube, J. E. 2011. Pretty, but dangerous! Records of non-native Monk Parakeet (Myiopsitta monachus) in Mexico. Revista Mexicana de Biodiversidad, 82: 1053-1056.

Martín, L. F. \& Bucher, E. H. 1993. Natal dispersal and first breeding age in Monk Parakeets. The Auk, 110: 930-933.

Minor, E. S., Appelt. C. W., Grabiner, S., Ward, L., Moreno, A. \& Pruett-Jones, S. 2012. Distribution of exotic Monk Parakeets across an urban landscape. Urban Ecosystems, 15: 979-991.

Mori, E., di Febbraro, M., Foresta, M., Melis, P., Romanazzi, E., Notari, A. \& Boggiano, F. 2013. Assessment of the current distribution of free-living parrots and parakeets (Aves: Psittaciformes) in Italy: a synthesis of published data and new records. Italian Journal of Zoology, 80: 158-167.

Mott, D. F. 1973. Monk Parakeet damage to crops in Uruguay and its control. Bird Control Seminars Proceedings, 102: 79-81.

Muñoz, A.R. \& Real, R. 2006. Assessing the potential range expansion of the exotic Monk Parakeet in Spain. Diversity and Distributions, 12: 656-665.

Navarro, J. L. \& Bucher, E. H. 1990. Growth of Monk Parakeets. Wilson Bulletin, 102: 520-525.

Navarro, J. L. \& Bucher, E.H. 1992. Annual variation in the timing of breeding of the Monk Parakeet in relation to climatic factors. Wilson Bulletin, 104: 545-549.

Navarro, J. L., Martela, M. B. \& Bucher, E. H. 1992. Breeding season and productivity of Monk Parakeets in Cordova, Argentina. Wilson Bulletin, 104: 413-424.

Pablo-López, R. A. 2009. Primer registro del perico argentino (Myiopsitta monachus) en Oaxaca, México. Huitzil, 10: 48-51.

Pajares, M. M. 2005. La cotorra argentina (Myiopsitta monachus) en la ciudad de Madrid, expansión y habitos de alimentación. Anuario Ornitológico de Madrid, 2005: 76-95.

Peris, S. J. \& Aramburú, R. M. 1995. Reproductive phenology and breeding success of the Monk Parakeet (Myiopsitta monachus monachus) in Argentina. Studies on Neotropical Fauna and Environment, 30: 115-119.

Pineda-López, R. \& Malagamba, A. 2011. Nuevos registros de aves exóticas en la ciudad de Querétaro, México. Huitzil, 12: 22-27.

Pruett-Jones, S., Newman, J. R., Newman, C. M., Avery, M. L. \& Lindsay, J. R. 2007. Population viability analysis of Monk Parakeets in the United States and examination of alternative management strategies. Human Wildlife Conflicts, 1: 35-44.

Ramírez-Alborez, J. E. 2012. Registro de la cotorra argentina (Myi- 
opsitta monachus) en la ciudad de México y áreas adyacentes. Huitzil, 13: 110-115.

Rodríguez-Pastor, R., Senar, J. C., Ortega, A., Faus, J., Uribe, F. \& Montalvo, T. 2012. Distribution patterns of invasive Monk Parakeets (Myiopsitta monachus) in an urban habitat. Animal Biodiversity and Conservation, 35: 107-117.

Ruiz-Corral, J. A., Medina-García, G., Meza-Sánchez, R., DíazPadilla, G. \& Serrano-Altamirano, B. 2006. Estadísticas climatológicas básicas del estado de Baja California Sur (Periodo 1961-2003). Libro técnico no. 2. INIFAP-CIRNO, Obregón, Sonora, México 268 pp.

Sol, D., Santos, D. M., Feria, E. \& Clavell, J. 1997. Habitat selection by the Monk Parakeet during colonization of a new area in Spain. The Condor, 99: 39-46.

Simberloff, D. 2003. How much information on population biology is needed to manage introduced species? Conservation Biology, 17: 83-92.

Simpson, M. B. \& Ruiz, R. C. 1974. Monk parakeets breeding in Buncombe County, North Carolina. Wilson Bulletin, 86: 171-172

Soto-Cruz, R. A., Lebgue-Keleng, T., Espinoza-Prieto, J. R., Quin- tana Martínez, R. M., Quintana-Martínez, G., Baderrama, S., Zamudio-Mondragón, F. R., Quintana-Chávez, M. A. \& Mondaca-Fernández, F. 2014. Primer registro de la cotorra Argentina (Myiopsitta monachus) en Chihuahua, México. Huitzil, 15: 1-5.

Strubbe, D. \& Matthysen, E. 2009. Establishment success of invasive ring-necked and Monk parakeets in Europe. Journal of Biogeography, 36: 2264-2278.

Tillman, E. A., Van Doom, A. \& Avery, M. L. 2000. Bird damage to tropical fruit in south Florida. Wildlife Damage Management Conferences, 13: 47-59.

Van Bael, S. \& Pruett-Jones, S. 1996. Exponential population growth of Monk parakeets in the United States. Wilson Bulletin, 108: 584588.

Vitouseck, P. M., Mooney, H. A., Lubchenco, J. \& Melillo, J. M. 1997. Human domination of earth's ecosystem. Science, 277: 494499.

Yoder, C. A., Avery, M. L., Keacher, K. L. \& Tillman, E. A. 2007. Use of DiazaCon ${ }^{\mathrm{TM}}$ as a reproductive inhibitor for monk parakeets (Myiopsitta monachus). Wildlife Research, 34: 8-13. 